\title{
Avaliação do desfecho dos conceptos com risco aumentado de ocorrência de anomalias cromossômicas calculado pela medida da translucência nucal
}

\author{
Outcome of fetuses with increased risk of chromosomal anomalies, based on \\ nuchal translucency measurement
}

Luiz Camano ${ }^{1}$, Antonio Fernandes Moron², Luciano Marcondes Nardozza ${ }^{3}$, David Baptista da Silva Pares ${ }^{4}$, Paulo Alexandre Chinen ${ }^{5}$

\section{RESUMO}

\begin{abstract}
Objetivo: avaliar o desfecho dos fetos que apresentam risco de anomalia cromossômica superior a 1:300, calculado pela medida da translucência nucal, por meio do programa da Fetal Medicine Foundation. Métodos: nas gestações únicas com risco para aneuploidia fetal superior a 1:300 foram avaliadas variáveis como: cariótipo fetal, abortamento espontâneo e provocado, prematuridade, óbito fetal, óbito neonatal, malformações estruturais e recém-nascidos normais. Usamos o teste exato de Fisher para fazer comparações de diferenças de proporções entre grupos. Resultados: foram observadas 193 (3,6\%) gestações únicas com risco de aneuploidia fetal acima de 1:300. Somente 165 gestações preencheram os critérios. Destas, apenas $32,1 \%$ foram submetidas a estudo do cariótipo fetal, com 8,5\% de anomalias cromossômicas $(85,7 \%$ de trissomia do cromossomo 21). Foram os seguintes os desfechos das gestações: $4,2 \%$ de abortos espontâneos, $4,2 \%$ de abortos induzidos, $4,8 \%$ de prematuridade, $1,8 \%$ de óbito neonatal, $1,8 \%$ de óbito fetal e $4,2 \%$ de malformações estruturais $(85,7 \%$ de malformações cardíacas). Aproximadamente $85,0 \%$ dos casos eram recém-nascidos normais. Pacientes com cariótipo anormal tiveram significativamente mais abortos induzidos $(p<0,001)$ e mais malformações $(p<0,001)$ que pacientes com cariótipo normal. Nenhum diagnóstico de doença gênica ou perda gestacional relacionada aos procedimentos invasivos foi detectado. Nos fetos com diagnostico no pré-natal de aneuploidia, a gestação foi interrompida em $66,7 \%$. Conclusões: a translucência nucal mantém seu papel no rastreamento das cromossomopatias, especialmente nas gestantes de baixo risco. Porém, o aconselhamento das gestantes com risco elevado deve ser prudente, uma vez que, apesar de estes casos apresentarem pior prognóstico fetal, a maioria apresenta desfecho favorável da gestação.
\end{abstract}

PALAVRAS-CHAVE: Aberrações cromossômicas; Transtornos cromossômicos; Prognóstico; Feto/ anormalidades; Rastreamento; Cariótipo

\section{ABSTRACT}

Purpose: to evaluate the outcome of fetuses with risk of chromosomal anomalies over 1:300, based on the nuchal translucency measurement, according to the Fetal Medicine Program. Methods: in the pregnancies with risk of chromosomal anomalies over 1:300, variables like fetal karyotype, spontaneous or induced abortion, prematurity, stillbirth, neonatal death, malformations, and healthy newborn were considered. We used Fisher's exact test to compare differences in proportions between groups. Results: we selected 193 (3.6\%) single pregnancies with risk of chromosomal anomalies over 1:300. Only 165 cases fulfilled the inclusion criteria. Of these only $32.1 \%$ underwent fetal karyotyping and of which $8.5 \%$ had chromosomal anomalies $(85.7 \%$ had trisomy 21$)$. Regarding pregnancy outcomes, $4.2 \%$ were spontaneous miscarriages, $4.2 \%$ induced abortions, $4.8 \%$ were premature, $1.8 \%$ had neonatal death, $1.8 \%$ were stillborn, and $4.2 \%$ had structural malformation $(85.7 \%$ congenital heart diseases). Almost $85.0 \%$ were healthy newborns. Patients with abnormal karyotyping had more induced abortions $(\mathrm{p}<0.001)$ and more structural malformations $(\mathrm{p}<0.001)$ than patients with normal karyotyping. None of the genetic diseases or miscarriages was associated with invasive procedures. Sixty-six percent of the pregnancies with prenatal

Departamento de Obstetrícia, Escola Paulista de Medicina da Universidade Federal de São Paulo - UNIFESP (SP).

1,2 Professor Titular.

3,4 Professor Assistente.

5 Pós-Graduando.

Correspondência: Paulo Alexandre Chinen

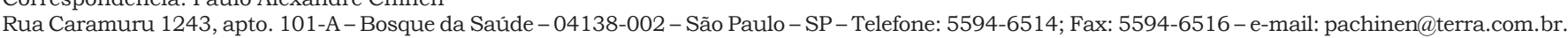

Recebido em: 26/3/2004

Aceito com modificações em: 28/3/2005 
diagnosis of abnormal karyotype were interrupted. Conclusion: nuchal translucency is an important screening tool for chromosomal diseases especially for low-risk pregnancies. However, counseling pregnancies with high risk of chromosomal anomalies should consider that, although these fetuses have a worse prognosis, most of the outcomes are favorable.

KEYWORDS: Chromossome aberrations; Chromossome disorders; Prognosis; Screening; Karyotype; Fetus/ abnormalities

\section{Introdução}

Durante a gestação os pais freqüentemente desejam saber se o seu filho tem ou terá alguma alteração anatômica, funcional, cognitiva ou genética. Em relação às alterações cromossômicas, se for considerado apenas o antecedente pessoal da gestante, serão identificados 3,0\% dos fetos com aneuploidia. Adotando a idade materna de 35 anos ou mais, para a indicação do estudo cromossômico fetal, o diagnóstico será feito em $30,0 \%$ dos $\operatorname{casos}^{1}$. Com o objetivo de melhorar a eficácia da detecção dos conceptos com doença cromossômica foram desenvolvidas outras técnicas. A ultra-sonografia trouxe a possibilidade de um novo método não invasivo que pudesse avaliar a anatomia fetal, os anexos, além da avaliação do líquido amniótico e do bem estar do concepto.

Em 1990, foi publicado o primeiro relato de edema nucal relacionado a alteração cromossômi$\mathrm{ca}^{2}$. Posteriormente, em 1992, foi definido o termo translucência nucal (TN) como o espaço preenchido por líquido entre o periósteo da coluna cervical e a pele fetal, sem septos em seu interior. Neste trabalho observou-se que nas gestações com espessura de TN superior a 3,0 mm, havia 35,0\% de alterações cromossômicas, em especial a sindrome de Down (SD), contrastando com 1,0\% no grupo controle ${ }^{3}$.

Outros autores ratificaram a TN como método adequado para o rastreamento da SD e de outras alterações do cariótipo fetal, utilizando como valores de corte números absolutos ou curvas de normalidade para a idade gestacional ${ }^{4}$. Além das anomalias cromossômicas, a TN alterada pode estar correlacionada com malformações estruturais, principalmente as cardiopatias ${ }^{5,6}$ e doenças gênicas.

No rastreamento das aneuploidias, quando é utilizada unicamente a TN, correlacionada ou não com a idade gestacional, são desconsideradas as variáveis maternas. Atentando para isso, foi desenvolvido um software que analisa conjuntamente valores de TN, idade gestacional, idade materna, antecedente de doença cromossômica e a presença de malformações estruturais no concepto. Assim sendo, foi definido que o risco maior ou igual a 1:300 selecionaria aproximadamente $82,2 \%$ dos fetos com SD, para uma taxa de falso-positivo de 5,0\%. Portanto, este ponto de corte poderia ser utilizado para oferecer o procedi- mento invasivo, buscando o diagnóstico das cromossomopatias com menores riscos ${ }^{7}$.

A maioria dos trabalhos relativos à TN tem como meta a sua eficácia no rastreamento das aneuploidias. São poucos aqueles que têm por objetivo acompanhar as gestações consideradas de risco elevado para aneuploidia seja pela medida da TN ou pelo risco corrigido (RC). Isto se dá pela alta freqüência de interrupções da gravidez, quando constatada ou mesmo na suspeita de anomalia cromossômica ou malformação fetal. Buscando melhorar o entendimento do desfecho dos conceptos com RC superior a 1:300, serão expostos os dados referentes à execução do estudo cromossômico, seu resultado e variáveis do período gestacional e pós-natal. O objetivo não será a avaliação da TN como método no rastreamento das aneuploidias, fato este já consolidado pela literatura nacional e internacional.

\section{Pacientes e Métodos}

Foram avaliadas pacientes encaminhadas à clínica privada, para realização de ultrasonografia obstétrica rotineira no primeiro trimestre da gravidez, no período compreendido entre agosto de 1995 e dezembro de 2002. Durante o exame foi executada a medida da TN, seguindo os critérios da Fetal Medicine Foundation 1994, retificados em $1999^{8}$. A via utilizada para medir a TN foi preferencialmente a transabdominal. Quando solicitado pelo médico assistente ou quando não foi possivel a medida pela via suprapúbica, seja pela posição fetal desfavorável, pelo biotipo da paciente ou pela incapacidade de repleção vesical adequada pela mesma, optou-se pelo exame transvaginal, após orientação e consentimento.

Os aparelhos utilizados foram o Toshiba AS - 340, Toshiba AS - 370 A e Medison 9900, com transdutores de 3,5 $\mathrm{MHz}$ e 5,0 MHz, pela via transvaginal e/ou abdominal, respectivamente, dependendo da necessidade.

Utilizou-se para o cálculo de risco individualizado para aneuploidia fetal o programa desenvolvido pela Fetal Medicine Foundation de Londres, versão 1995. Tal programa levou em conta para o cálculo do RC e individualizado para cada paciente os seguintes parâmetros: idade materna, histórico 
individual de aneuploidia, observação de malformações estruturais, idade gestacional baseada no comprimento cabeça-nádega e o valor da TN.

A amostra deste estudo foi composta por pacientes com gestações únicas, nas quais o $\mathrm{RC}$ foi superior a 1:300 e cujo resultado do desfecho pré e/ou pós-natal era conhecido.

Assim sendo, das 5.351 ultra-sonografias com mensuração de TN, 193 (3,6\%) apresentavam o RC superior a 1:300. Estas pacientes estavam sendo acompanhadas por 118 diferentes obstetras. Das 193 gestações, foram obtidas as informações referentes ao desfecho da gestação em 165 (85,5\%), determinando a amostra do presente estudo.

Os dados avaliados foram obtidos por meio de consulta junto aos médicos assistentes, às próprias pacientes e/ou prontuários médicos. Os $14,5 \%$ de perdas de resultado foram decorrentes da não resposta ao questionário, perda do acompanhamento prénatal pelo médico assistente ou pela não localização da paciente com os dados cadastrais disponiveis. As variáveis do período gestacional e pós-natal avaliadas foram: abortamento espontâneo ou provocado; prematuridade, óbito fetal, óbito neonatal; presença de malformações estruturais diagnosticadas ao nascimento (ME) e recém-nascido normal. Deve ser salientado que para a melhor avaliação dos dados, foi considerada ME toda aquela visivel ao nascimento e/ou aquela diagnosticada após investigação de achado anormal no exame físico, durante a estada no berçário. Os achados fenotípicos inespecíficos (exemplo: alterações de pregas palmares, face sindrômica) não foram considerados ME. Foi considerado recém-nascido normal todo aquele nascido vivo, com estudo cromossômico dentro da normalidade durante a gestação, sem ME aparente ou aquele sem avaliação do cariótipo no período antenatal, que não apresentava malformação ou sinais que justificassem o estudo genético no berçário. Os dados do período pós-natal são restritos ao tempo de internação no berçário.

A indicação da avaliação do cariótipo fetal e da via de coleta do material foi feita pelo médico assistente da paciente. Nas 165 gestações estudadas, foram realizados $53(32,1 \%)$ exames de cariótipo do produto conceptual, sendo o diagnóstico feito por biópsia de vilosidade coriônica em 16 $(30,2 \%)$, por amniocentese em $31(58,5 \%)$, por análise de material de aborto em $1(1,9 \%)$ e em $5(9,6 \%)$ casos por pesquisa pós-natal devido a alterações fenotípicas do recém-nascido.

Os 165 casos foram divididos em dois grupos: o grupo cariótipo positivo (GCP) foi composto pelas gestantes cujo cálculo de risco para aneuploidias foi superior a 1:300 e que realizaram estudo cromossômico, pré ou pós-natal, com alteração no cariótipo do produto conceptual. O grupo cariótipo negativo (GCN) foi composto pelas gestantes cujo cálculo de risco para aneuploidias foi superior a 1:300 e que realizaram estudo cromossômico sem alteração no cariótipo fetal ou aquelas sem estudo genético no periodo pré-natal e para as quais não foi necessária a realização do mesmo no pós-parto.

Os objetivos e a estruturação do trabalho foram aprovados pelo Comitê de Ética Médica em Pesquisa da Universidade Federal de São Paulo / Escola Paulista de Medicina.

\section{Análise estatistica}

Para comparações de dados categóricos foi utilizado o teste exato de Fisher, visto que o valor esperado foi inferior a cinco em todas as tabelas de contingência.

Diferenças entre médias de dados contínuos foram testadas utilizando-se testes paramétricos e não paramétricos, que, sem exceção, mostraram resultados similares. Somente os resultados dos testes paramétricos serão mostrados. Foi utilizado o teste $t$ de Student.

A probabilidade (p) menor que 0,05 foi considerada para indicar significância estatística. Todos os testes foram bicaudados. Intervalo de confiança (IC) de $95 \%$ foi calculado em relação às diferenças entre médias. Toda a análise foi calculada segundo o pacote estatístico SPSS (Statistical Package for the Social Sciences) 11.5.1 para Windows.

\section{Resultados}

Foram revistos 5.531 laudos de ultrasonografia. Destes, 165 preenchiam todos critérios de inclusão. Não houve diferenças significantes entre o GCN e o GCP em relação aos valores idade materna $(38,7 \pm 3,9$ vs $37,4 \pm 4,9$, IC $95 \%$ : $-0,9$ a 3,5 , $\mathrm{t}(163)=1,2, \mathrm{p}=0,247)$ e idade gestacional, calculada pelo comprimento cabeça-nádega do concepto $(62,4 \pm 10,6$ vs $62,5 \pm 10,0$, IC $95 \%:-6,0$ a $5,8, t(163)=$ $-0,1, p=0,975)$. Porém, existiram diferenças significantes quanto aos valores das TN $(2,2 \pm 1,0$ vs $3,7 \pm 1,8$, IC 95\%: $-2,1$ a $-0,9, \mathrm{t}(163)=-4,9, \mathrm{p}<0,001)$ e aos denominadores dos RC $(165,6 \pm 84,5$ vs $57,6 \pm 80,1$, IC 95\%: 61,6 a 154,5, t(163) $=4,6, \mathrm{p}<0,001)$.

Em $39(23,6 \%)$ casos o cariótipo foi realizado e considerado normal. Nas $14(8,5 \%)$ gestações com alteração cromossômica, observamos $12(85,7 \%)$ casos de trissomia do cromossomo $21,1(7,1 \%)$ caso de monossomia X $(45, \mathrm{X0})$ e $1(7,1 \%)$ caso de triploidia (69, XXX). Em $112(67,9 \%)$ pacientes não foi solicitada a avaliação cromossômica durante a gestação ou após o nascimento, por opção da paciente e/ou do médico assistente. Não foi observado nenhum caso de perda gestacional que pudesse ser relacionada aos procedimentos invasivos. 
Os desfechos das 165 gestações serão analisados no que concerne às variáveis abortamento espontâneo, abortamento provocado, prematuridade, óbito fetal e óbito neonatal. As freqüências demonstradas na Tabela 1 são relativas à presença destas variáveis durante o periodo estudado, sendo que em alguns casos houve sobreposição de ocorrências como prematuridade e óbito neonatal.

Estudando os achados em separado temos que, em relação ao abortamento espontâneo, embora a freqüência de perdas gestacionais tenha sido quase cinco vezes maior no GCP $(14,3 \%)$ quando comparado ao GCN $(3,6 \%)$, não foram observadas diferenças significantes entre os grupos (teste exato de Fisher, $p=0,110$ ). Porém, quando avaliado o abortamento provocado $(1,8 \%)$, foram notados seis casos no GCP e um no GCN. No estudo desta variável, houve diferença significante entre os grupos (43 vs $1 \%$, teste exato de Fisher, $\mathrm{p}<0,001)$. Deve ser ressaltado que dos 14 casos com diagnóstico de aneuploidia fetal, cinco tiveram a análise cromossômica realizada após o nascimento. Sendo assim, dos nove casos com confirmação da anomalia cromossômica no período antenatal, $66,7 \%$ dos casais optaram pela interrupção da gestação.

Tabela 1 - Distribuição dos desfechos das 165 gestações com risco aumentado de ocorrência de anomalia cromossômica fetal, calculado pela translucência nucal.

\begin{tabular}{lccccccc}
\hline Grupo & AE & AP & Pt & OF & ON & ME & RNN \\
\hline GCP & 2 & 6 & 0 & 1 & 0 & 4 & 0 \\
$\mathrm{n}=14$ & $1,2 \%$ & $3,6 \%$ & $0 \%$ & $0,6 \%$ & $0 \%$ & $2,4 \%$ & $0 \%$ \\
GCN & 5 & 1 & 8 & 2 & 3 & 3 & 140 \\
$\mathrm{n}=151$ & $3,0 \%$ & $0,6 \%$ & $4,8 \%$ & $1,2 \%$ & $1,8 \%$ & $1,8 \%$ & $84,8 \%$ \\
Total & 7 & 7 & 8 & 3 & 3 & 7 & 140 \\
$\mathrm{n}=165$ & $4,2 \%$ & $4,2 \%$ & $4,8 \%$ & $1,8 \%$ & $1,8 \%$ & $4,2 \%$ & $84,8 \%$
\end{tabular}

$\mathrm{AE}=$ abortamento espontâneo; $\mathrm{AP}=$ abortamento provocado; $\mathrm{Pt}=$ prematuridade; $\mathrm{OF}=$ óbito fetal; $\mathrm{ON}=$ óbito neonatal; $\mathrm{ME}$ = presença de malformações estruturais diagnosticadas ao nascimento; RNN = recém-nascido normal; GCP = grupo cariótipo positivo; $G C N$ = grupo cariótipo negativo.

Nas análises a seguir, os casos de abortamento serão excluídos, procurando tornar mais fidedigna a história natural destas gestações após a segunda metade da gravidez. Portanto, serão analisadas 145 pacientes do GCN e seis do GCP. No que tange à prematuridade, não foi constatada diferença significante entre o GCN e o GCP (5 vs 0\%, teste exato de Fisher, $p=0,717)$. A moléstia hipertensiva específica da gravidez foi relatada como fator causal em cinco dos oito casos de prematuridade.

Quanto ao óbito fetal, não foi observada diferença significante entre o GCP e o GCN (17 vs 1\%; teste exato de Fisher, $p=0,115$ ). O único óbito fetal no GCP foi de um concepto com trissomia do cromossomo 21, com análise morfológica pela ultrasonografia adequada, no qual não foi realizada necrópsia. Os dois eventos do GCN foram relacio- nados respectivamente com nó verdadeiro de cordão e descolamento prematuro da placenta. Ambos não realizaram estudo cromossômico.

Não houve diferença entre os grupos no que se refere à variável óbito neonatal $(2 \%$ no GCN e $0 \%$ no GCP, teste exato de Fisher, $p=0,885$ ). Nos três casos de óbito neonatal, a prematuridade foi determinante em dois deles, enquanto a cardiopatia ocorreu no outro caso.

Avaliando a variável malformação estrutural, notou-se diferença significante nas proporções entre o GCP e o GCN (67 vs 2\%, teste exato de Fisher, $p<0,001)$. Das anomalias observadas no GCP, quatro foram cardiopatias, ao passo que das do GCN, duas foram cardiopatias e uma foi pé torto.

Analisando os 140 recém-nascidos considerados normais, $38(27,1 \%)$ haviam feito análise do cariótipo. Obviamente não foram relacionados recém-nascidos normais no GCP.

Assim sendo, 84,8\% dos recém-nascidos com $\mathrm{RC}$ acima de 1:300 não apresentavam anomalias cromossômicas ou malformações diagnosticadas durante a estada no berçário, e 78,2\%, além das afirmações anteriores, não tiveram qualquer dissabor até o final da gravidez ou período neonatal.

Não foram descritas doenças gênicas, provavelmente pelo curto período de observação das crianças após o nascimento.

\section{Discussão}

Em estudo multicêntrico, a ocorrência de RC superior a 1:300 foi de $8,8 \%$, com valor preditivo positivo para o rastreamento de aneuploidias de $6,2 \%^{7}$. Neste trabalho, os respectivos valores foram 3,6 e $8,5 \%$.

Das gestações com diagnóstico de anomalia cromossômica, $85,8 \%$ eram trissomia do cromossomo 21 . As outras aneuploidias encontradas foram a monossomia do cromossomo $\mathrm{X}$ e a triploidia. Estes dados são coerentes com a literatura, na qual existe sempre o predomínio, mas não a exclusividade, da SD após o rastreamento positivo pela $\mathrm{TN}^{8-10}$.

Em $112(67,9 \%)$ casos não foi solicitado o estudo cromossômico do produto conceptual. Um dos principais motivos para isto foi a não-indicação da propedêutica invasiva pelo profissional assistente após avaliação do risco-benefício do procedimento. O segundo fator de destaque foi a opção tomada pelo casal pela não-realização da avaliação do cariótipo fetal, visto que a gravidez prosseguiria independentemente do resultado do cariótipo ou por acharem significativo o risco da perda da gestação relacionado à coleta do material. 
Os resultados estatísticos demonstram que os dois grupos são homogêneos quanto a distribuição das idades maternas e idades gestacionais. $\mathrm{O}$ fator determinante na diferenciação entre eles foi a medida da TN, que produz uma relação diretamente proporcional ao risco para alterações no padrão dos cariótipos fetais.

Se fosse realizada a indicação de estudo cromossômico baseada apenas na idade materna acima de 35 anos, $28,6 \%$ das gestações com aneuploidia não seriam diagnosticadas durante o pré-natal. Ao passo que, se o parâmetro utilizado fosse a medida absoluta da TN acima de $2,5 \mathrm{~mm}$, não seriam detectados $14,3 \%$ dos casos. Desta forma, destaca-se a importância do uso de programa de cálculo de risco para aneuploidia do concepto como o da Fetal Medicine Foundation, individualizando a indicação do estudo do cariótipo fetal.

Abaixo seguem as considerações sobre as variáveis do desfecho das gestações em questão. Deve-se ressaltar que as publicações nacionais e internacionais utilizam pontos de corte variados, o que pode interferir na análise dos dados.

Avaliando a variável aborto espontâneo, não se encontra diferença estatística entre os dois grupos $(\mathrm{p}=0,110)$. Na presente amostra identificou-se $4,2 \%$ de perda espontânea, sendo que $28,6 \%$ tinham alteração do cariótipo. O valor total de perdas espontâneas não ultrapassa os 15,0\% de abortamento espontâneo clinicamente diagnosticados na população geral, da mesma maneira quando comparados com a literatura ${ }^{11,12}$, incluindo os fetos com aneuploidias ${ }^{13}$. Sabe-se que a perda gestacional espontânea acaba por funcionar como seleção natural dos conceptos acometidos por defeitos cromossômicos. Nestes casos, entre a $12^{\text {a }}$ semana de gestação e o termo ocorre $30,0 \%$ de perda gestacional, sendo que mais $10 \%$ abortará até a $16^{\mathrm{a}}$ semana de gestação ${ }^{8}$.

Quando estudada a variável aborto provocado, a freqüência neste estudo foi de $4,2 \%$, apresentando diferença estatística entre os grupos $(p<0,001)$, com predomínio no GCP. Levando em conta apenas os casos com diagnóstico pré-natal de aneuploidia, $66,7 \%$ interromperam a gestação. Estes dados são elevados, principalmente se for levado em conta que este é procedimento que necessita de autorização judicial e que $100,0 \%$ destes casos eram trissomia do cromossomo 21 , aneuploidia compativel com a vida. A incidência de abortamentos provocados foi compativel com a literatura internacional ${ }^{14,15}$, com a ressalva que nos centros onde foram realizados tais estudos, a prática é legalmente aceita e denominada interrupção médica da gravidez.

A análise das outras variáveis do desfecho gestacional será feita seguir e devemos relembrar que foram excluídos os casos de abortamento da análise estatística. Deve-se levar em consideração que as interrupções intencionais da gestação no primeiro trimestre podem ter interferido no curso natural das gravidezes, funcionando como viés no estudo das variáveis.

No que concerne à variável prematuridade, não houve diferença significante entre os grupos $(p=0,717)$. Os $4,8 \%$ de prematuridade são semelhantes ao dado internacional disponivel, em que foi utilizada medida da TN isoladamente ${ }^{16}$. A hipertensão materna foi causa de $62,5 \%$ dos partos prematuros, sendo que a idade materna destas mães foi de 41 anos. Sendo assim, a idade materna pode ser interpretada como fator contribuinte para o aumento do RC, da mesma forma que aumenta a possibilidade de doenças sistêmicas associadas ao período gravídico-puerperal, como a hipertensão e o diabete gestacional.

Para a variável óbito fetal também não foi constatada diferença estatística entre as amostras $(p=0,115)$. No total da amostra obteve-se $1,8 \%$ de óbito fetal. Considerando somente as 148 gestações que chegaram ao fim, observou-se 2,0\% de óbito fetal, próximo aos valores apresentados por outros autores, que utilizaram curvas de normalidade ou valores fixos de TN como ponto de corte ${ }^{11,13}$. Nos casos com cariótipo normal, as etiologias foram o nó verdadeiro de cordão umbilical e a moléstia hipertensiva da gestação numa gestante de 38 anos. Aparentemente apenas a idade materna poderia justificar um fator de risco comum entre o óbito fetal e o RC aumentado. No caso com aneuploidia, não houve comprovação do fator causal.

Diante do óbito neonatal não se obteve diferença significativa entre os grupos $(p=0,885)$. A freqüência de 1,8\% de óbito neonatal vai ao encontro da literatura ${ }^{12,14,16}$. Como era de se esperar, a prematuridade foi fator associado à neomortalidade em dois terços dos casos.

$\mathrm{Na}$ amostra estudada notou-se 3,6\% de malformações estruturais. A estatística evidenciou diferença significante entre os grupos $(p<0,001)$, com maior predisposição no fetos com alterações cromossômicas. Das ME detectadas, 83,3\% foram cardiopatias congênitas, sendo que no GCP a freqüência foi de $100 \%$. Estes dados ratificam que TN alterada tem boa correlação com alterações cardiacas fetais, da mesma forma que as aneuploidias $^{5,6}$. Na vigência de TN alterada e cariótipo normal faz-se necessária a realização de ecocardiografia fetal. Os dados relacionados à incidência de $\mathrm{ME}$ nas gestações com TN alterada variam de 6,3 a $26,1 \%$ em conceptos euplóides ${ }^{12,17}$.

Direcionando a análise estatística aos recém-nascidos normais, foi obtida diferença estatística entre os grupos $(p<0,001)$. Certamente não foram observados recém-nascidos normais no GCP. Dos recém-nascidos, 84,8\% não tinham qualquer 
alteração cromossômica ou estrutural no período do estudo. Fazendo um paralelo com a literatura disponivel, os valores estão próximos às casuísticas que utilizaram como pontos de corte valor fixo $(3,0$ $\mathrm{mm}$ ) ou curvas de normalidade de $\mathrm{TN}^{11,12,16}$.

Não obtivemos relatos de doenças gênicas em nossa amostra. Nos trabalhos que citam este tipo de doença, o acompanhamento pré-natal ocorre no mesmo centro onde é feita a puericultura, facilitando o acompanhamento longitudinal das crianças e o intercâmbio entre as especialidades. Devemos lembrar que o período de avaliação foi restrito à estada do recém-nato no berçário. Também não foi relatado antecedente obstétrico de cromossomopatia na nossa amostra.

Observa-se que em 129 (78,2\%) gestações não houve alteração cromossômica ou qualquer outro dissabor até o final da gravidez e no período neonatal. Concentrando a análise na alteração cromossômica, em 137 (83,0\%) recém-nascidos saudáveis, o estudo do cariótipo fetal seria dispensável, deixando de correr o risco da perda teórica de aproximadamente 1,5 gestação de cariótipo normal por intercorrências do procedimento na amostra estudada.

Desta maneira, ratificamos que a TN é um exame de rastreamento e que deve ser associada a programas de cálculo de risco como o da Fetal Medicine Foundation, individualizando o resultado e a orientação da gestante.

$\mathrm{O}$ aconselhamento das gestantes com probabilidade para cromossomopatia fetal superior a $1: 300$, calculado pela TN, deve ser cauteloso e baseado em múltiplos parâmetros. Não foi observada anomalia cromossômica ou desfecho desfavorável da gestação em $78,2 \%$ dos nossos casos. É importante ressaltar que a TN mantém seu lugar no rastreamento das alterações do cariótipo na população geral. Porém, seu valor preditivo positivo inferior a 10,0\% nos faz sugerir que sejam associados outros métodos, como a análise bioquímica, buscando diminuir os falso-positivos.

\section{Referências}

1. Zeitune M, Aitken DA, Crossley JA, Yates JR, Cooke A, Ferguson-Smith MA. Estimating the risk of a fetal autosomal trisomy at mid-trimester using maternal serum alpha-feto protein and age: a retrospective study of 142 pregnancies. Prenat Diagn. 1991;11(11):847-57.

2. Szabo J, Gellen J. Nuchal fluid accumulation in trisomy-21 detected by vaginosonography in first trimester. Lancet. 1990;336(8723):1133.

3. Nicolaides KH, Azar G, Byrne D, Mansur C, Marks K. Fetal nuchal translucency: ultrasound screening for chromosomal defects in first trimester of pregnancy. BMJ. 1992;304(6831):867-9.
4. Nicolaides KH. Screening for chromosomal defects. Ultrasound Obstet Gynecol. 2003;21(4):313-21.

5. Hyett J, Perdu M, Sharland GK, Snijders RS, Nicolaides $\mathrm{KH}$. Increased nuchal translucency at 10-14 weeks of gestation as a marker for major cardiac defects. Ultrasound Obstet Gynecol. 1997;10(4):242-6.

6. Hyett J, Moscoso G, Nicolaides K. Abnormalities of the heart and great arteries in first trimester chromosomally abnormal fetuses. Am J Med Gen. 1997;69(2):207-16.

7. Snijders RJ, Noble P, Sebire N, Souka A, Nicolaides $\mathrm{KH}$. UK multicentre project on assessment of risk of trisomy 21 by maternal age and fetal nuchal-translucency thickness at 10-14 weeks of gestation. Lancet. 1998;352(9125):343-6.

8. Nicolaides KH, Sebire N, Snijders RJ. The 11-14 week scan. The diagnosis of fetal abnormalities. London: Parthenon; 1999. p. 15.

9. Nicolaides KH, Brizot ML, Snijders RJ. Fetal nuchal translucency: ultrasound screening for fetal trisomy in first trimester of pregnancy. Br J Obstet Gynaecol. 1994;101(9):782-6.

10. Snijders RJ, Sebire NJ, Nayar R, Souka A, Nicolaides $\mathrm{KH}$. Increased nuchal translucency in trisomy 13 fetuses at 10-14 weeks of gestation. Am J Med Gen. 1999;86(3):205-7

11. Michailidis GD, Economides DL. Nuchal translucency measurement and pregnancy outcome in karyotypically normal fetuses. Ultrasound Obstet Gynecol. 2001;17(2):102-5.

12. Souka AP, Krampl E, Bakalis S, Heath V, Nicolaides $\mathrm{KH}$. Outcome of pregnancy in chromosomally fetuses with increased nuchal translucency in the first trimester. Ultrasound Obstet Gynecol. 2001;18(1):9-17.

13. Bilardo CM, Pajkrt E, de Graaf I, Mol BW, Bleker OP. Outcome of fetuses with enlarged nuchal translucency and normal karyotype. Ultrasound Obstet Gynecol. 1998;11(6):401-6.

14. Adekunle O, Gopee A, el-Sayed M, Thilaganathan B. Increased first trimester nuchal translucency: pregnancy and infant outcomes after routine screening for Down's syndrome in an unselected antenatal population. Br J Radiol. 1999;72(857):457-60.

15. Maymon R, Jauniaux E, Cohen O, Dreazen E, Weinraub $Z$, Herman A. Pregnancy outcome and infant follow-up of fetuses with abnormally increased first trimester nuchal translucency. Hum Reprod. 2000;15(9):2023-7.

16. Pajkrt E, Mol BW, Bleker OP, Bilardo CM. Pregnancy outcome and nuchal translucency measurements in fetuses with a normal karyotype. Prenat Diagn. 1999;19(12):1104-8.

17. Brady AF, Pandya PP, Yuksel B, Greenough A, Patton MA, Nicholaides KH. Outcome of cromosomally normal livebirths with increased fetal nuchal translucency at 10-14 weeks' gestation. J Med Genet. 1998;35(3):222-4. 